\title{
Urgences
}

\section{La genèse de " Julius " : Au commencement était le chaos}

\section{Chantal Bouchard}

Numéro 24, juillet 1989

Le manuscrit sous l'angle

URI : https://id.erudit.org/iderudit/025530ar

DOI : https://doi.org/10.7202/025530ar

Aller au sommaire du numéro

Éditeur(s)

Urgences

ISSN

0226-9554 (imprimé)

1927-3924 (numérique)

Découvrir la revue

Citer cet article

Bouchard, C. (1989). La genèse de " Julius " : Au commencement était le chaos. Urgences, (24), 35-42. https://doi.org/10.7202/025530ar d'utilisation que vous pouvez consulter en ligne.

https://apropos.erudit.org/fr/usagers/politique-dutilisation/ 


\section{La genèse de "Julius»: Au commencement était le chaos \\ Chantal Bouchard, Université McGill}

Si je me permets, dans l'introduction de mon exposé, cette citation biblique, c'est qu'elle se présente à mon esprit à cause de la métaphore qui fonde l'utilisation du mot genèse en analyse littéraire. Sans doute les premiers critiques à en faire usage ontils éprouvé comme moi, devant le monceau de brouillons, d'ébauches, de textes parallèles et de versions constituant la matière originelle, une certaine angoisse. Retracer la genèse d'une œuvre passe donc nécessairement par une étape fondamentale: mettre de l'ordre. Quels sont les critères qui permettront le classement des textes? II faut faire feu de tout bois et, en ce domaine, les indices matériels et les critères textuels sont complémentaires. Plus le dossier est abondant, plus l'analyse devra être serrée.

Lorsque nous avons commencé, Nicole Deschamps et moi, l'édition critique des nouvelles d'Alain Grandbois, qui sera publiée dans la collection "Bibliothèque du nouveau monde", l'une de nos premières décisions a été de remettre le dossier de la nouvelle intitulée "Julius" à plus tard, tant il nous paraissait épineux. Ce n'est qu'après nous être longuement familiarisées avec les manuscrits et les méthodes de Grandbois que nous nous sommes attaquées à ce dernier pic.

Il convient, je crois, pour vous permettre de bien comprendre les problèmes soulevés par l'édition de cette nouvelle, que je vous en fasse un bref résumé descriptif.

"Julius" est une des nouvelles les plus courtes du recueil Avant le chaos ${ }^{1}$; elle fait à peine une quinzaine de pages dans l'édition format livre de poche de HMH datant de 1964. Elle est entièrement constituée d'une seule longue phrase rythmée par des virgules et des tirets, l'unique point étant le point final. Le narrateur se situe une trentaine d'années après les événements, racontant au passé les aventures d'un jeune homme appelé

1 Alain Grandbois, «Julius», Avant le chaos [1945], coll. «L'Arbre», Montréal, HMH, 1964, p. 227-241. 
Julius qu'il présente comme son inséparable ami de l'époque. Après une longue introduction dans laquelle le narrateur décrit ironiquement son époque, il présente «la vie d'un jeune homme lancé dans le siècle» (p. 227) avant la Seconde Guerre mondiale.

Julius et le narrateur sont des amis très proches, menant une vie insouciante et aisée sur la Cóte d'Azur dans les années trente. Un soir, Julius entre au casino de Juan-les-Pins, où le barman qu'il connaît bien lui confie la garde d'une fort belle dame évanouie, le temps d'aller chercher de l'aide. Le lendemain matin, Julius part pour Paris où il reste une semaine, ignorant que la veille, la dame a déclaré le vol de sa bague sertie d'un diamant fabuleux. La police soupçonne Julius, puis classe l'affaire, la bague perdue ayant été retrouvée. Au retour du jeune homme à Juan-les-Pins, le barman lui raconte toute l'histoire et Julius est très blessé d'avoir été soupçonné de vol. Plus tard, il retrouve la dame au diamant dans une soirée. Elle lui offre son amour qu'il refuse. Quelques années après, il revient sur la cóte en compagnie d'une belle femme qu'il vient d'épouser à Paris. Ils vivent quelque temps un amour légendaire dont le narrateur est témoin, puis un soir, ils s'embarquent sur un yacht pour une longue croisière. En quittant la rade, le bateau explose "et ce fut la fin [...] du plus bel amour du monde" (p. 241).

Revenons à notre chaos originel qui se présentait, au commencement, sous la forme d'un lourd dossier dans lequel nous avions regroupé tous les textes manuscrits ou dactylographiés qui semblaient pertinents. Tous ceux contenant le nom de Julius ou une histoire semblable à l'anecdote de la nouvelle et que nous avions repérés au fil de nos recherches dans les archives avaient donc été retenus. Au total, et je le rappelle, pour une nouvelle qui éditée fait une quinzaine de pages, nous nous trouvions devant trois cent huit feuillets dont la plupart sont manuscrits. Je vous épargnerai la description du long travail qui fut nécessaire pour mettre un peu d'ordre dans tout cela. Disons simplement qu'une fois les incomplets regroupés, nous nous retrouvions avec dix-huit manuscrits dont l'état variait du brouillon griffonné au texte achevé, en plus, bien entendu, des deux versions éditées de la nouvelle.

Parmi les manuscrits, il n'y en a que deux qui soient de véritables versions de la nouvelle définitive. Deux versions très proches l'une de l'autre, puisqu'il s'agit d'un manuscrit dactylographié de dix-huit feuillets et de sa copie au carbone, tous deux sous le titre "Julius", et qui ne se différencient que 
par quelques corrections ou ajouts. Le premier de ces textes a dû servir pour la première édition de la nouvelle, car il comporte l'ajout suivant: par Alain Grandbois de l'Académie canadiennefrançaise, et quelques indications typographiques. ${ }^{2}$

Même si les seize autres textes sont d'une rédaction trop éloignée pour être considérés comme des versions de la nouvelle éditée, quatre d'entre eux présentent assez d'analogies avec elle sur le plan de l'anecdote pour qu'on y voie des ébauches ou, si l'on veut, des versions antérieures. Le manuscrit sans titre ["Heureuse jeunesse..."] contient la même anecdote avec quelques écarts et il est rédigé comme la nouvelle, c'est-à-dire sans ponctuation. ${ }^{3}$

Les deux textes suivants, un manuscrit dactylographié de trois pages et sa copie au carbone, intitulés' "Le diamant", donnent une ébauche des premières pages, soit l'introduction de la nouvelle; ils sont également rédigés sans ponctuation. ${ }^{4}$

Enfin, un manuscrit de vingt-huit feuillets, intitulé lui aussi "Le diamant", a dû être reconstitué, car la pagination en est anarchique, ce qui est fréquent chez Grandbois, et le papier varie en formats et en couleurs. Le déroulement du texte a permis de rattacher les liasses. Ce texte présente toujours l'anecdote du diamant volé, notre fil d'Ariane comme on le verra plus loin, mais il est écrit sous une forme plutôt autobiographique, l'aventure arrivant à l'auteur lui-même, et il a une ponctuation normale contrairement aux textes précédents. ${ }^{5}$

$2 \alpha$ Julius», manuscrit dactylographié de 18 feuillets $(215 \mathrm{~mm} \times 278$ $\mathrm{mm}$ ), corrections à l'encre bleue et au crayon bleu, indications typographiques au crayon rouge. aJulius», copie au carbone du précédent, corrections à l'encre bleue, les pages 13 et 15 manquent. Fonds Grandbois, Bibliothèque nationale du Québec, boîte 3 , chemise 10. La première édition de eJulius a paru dans les Cahiers de l'Académie canadienne-française, coll. «Contes et nouvelles", no 4, Montréal, 1959, p. 57-67.

3 [«Heureuse jeunesse..."], manuscrit de 20 feuillets (127 $\mathrm{mm} \times 202$ $\mathrm{mm}$ ), paginés 39-59, sans titre, encre bleue. Fonds Grandbois, BNQ, boîte 3, chemise 10.

4 *Le diamant*, manuscrit dactylographié de 3 feuillets $(215 \mathrm{~mm} \times$ $278 \mathrm{~mm}$ ), corrections à l'encre bleue. «Le diamant », copie au carbone du précédent, corrections à l'encre bleue. Fonds Grandbois, BNQ, boîte 3, chemise 10.

5 "Le diamant», manuscrit de 28 feuillets; 5 feuillets $(124 \mathrm{~mm} \times 203$ $\mathrm{mm}$ ) paginés I-V, suivis de 23 feuillets (127 mm x $203 \mathrm{~mm}$ ); papier vert, 
Les sept textes suivants se rattachent à la nouvelle de manière plus lâche, quoique réelle. Plusieurs sont écrits sans ponctuation, on y retrouve le personnage de Julius, alter ego de l'auteur, jeune poète voyageur faisant la noce à Paris, à Cannes, ou méme à Montréal. Cependant, on ne trouve plus dans ces textes l'anecdote du diamant qui est le centre de la nouvelle; le héros y vit d'autres aventures. ${ }^{6}$

Un autre groupe de manuscrits présente une prose poétique très éloignée de la nouvelle, le seul lien étant le nom du personnage, Julius. ${ }^{7}$

Enfin, un poème intitulé «Enfer-ciel", où il est également question de Julius, clôt l'abondant dossier. 8

Une fois cet inventaire réalisé, il convenait de découvrir, dans la mesure du possible, quels rapports, disons, génétiques, ces textes avaient entre eux et dans quel ordre ils avaient pu être rédigés. En partant de la version éditée, le cas des deux premiers textes, soit les manuscrits dactylographiés, fut vite réglé, puisqu'il s'agissait du même texte que celui de la nouvelle éditée, avec seulement quelques variantes. Pour les quatre textes suivants, que nous appelons des versions antérieures, la question devenait plus épineuse. Deux éléments nous

paginés IV-IX (VII/7, VIII/8, IX/9) et 10-27, crayon à mine. Fonds Grandbois, BNQ, boîte 3, chemise 10.

6 "Le diamant , manuscrit de 33 feuillets; [ $\propto$ Un matin à Cannes... $»$ ], manuscrit sans titre de 8 feuillets; «Julius", manuscrit de 73 fevillets. Fonds Grandbois, BNQ, boîte 3, chemise 10. ["Tu as giflé Bernard...»], manuscrit sans titre de 23 feuillets. Fonds Grandbois, BNQ, boîte 3 , chemise 16. aJulius et Madeleinex, manuscrit de 15 feuillets. Fonds Grandbois, boîte 3, chemise 9; le texte commence sur la page 10 d'un

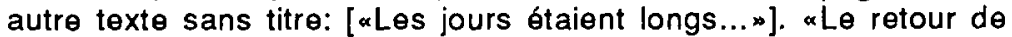
Julius», manuscrit de 6 feuillets; «L'Argent», manuscrit de 2 feuillets. Fonds Grandbois, boîte 3, chemise 10.

7 aDélivrance du jourm, sous ce titre, manuscrits de 5 feuillets, 10 feuillets, 12 feuillets, 2 feuillets et 2 feuillets. Fonds Grandbois, BNQ, boîte 3, chemise 14. Ces textes ont été édités sous le titre: Délivrance du jour et autres inédits, Montréal, éditions du Sentier, 1980, p.79. [ $\times$ Je puis affirmer...»], manuscrit sans titre de 7 feuillets; [ $\alpha$ Julius: les fontaines...»], manuscrit sans titre de 4 feuillets. Fonds Grandbois, BNQ, boîte 3, chemise 10. [«Les jours étaient longs...»], manuscrit sans titre de 10 feuillets, Fonds Grandbois, BNQ, boitte 3, chemise 9. 8 «Enfer-cielø, manuscrit de 6 feuillets. Fonds Grandbois, BNQ, boite 1, chemise 5 . 
permettaient de supposer un ordre: le fil narratif et le style. Entre [ "Heureuse jeunesse..."] et "Le diamant" de vingt-huit feuillets, le choix était clair. ["Heureuse jeunesse..."] présente, à peu de chose près, la même histoire que la nouvelle, et ce, sans ponctuation, alors que "Le diamant" présente lui l'aventure du diamant disparu comme un récit autobiographique; le personnage de Julius n'y figure pas et le style est plus conventionnel au chapitre de la ponctuation. [Heureuse jeunesse..."] est donc postérieur au "Diamant". Là où les choses se compliquent singulièrement, c'est que le personnage de Julius et le style sans ponctuation apparaissent dans toute une série d'autres textes où, par contre, l'anecdote est tout à fait différente.

Ayant présenté ailleurs l'analyse des éléments qui permettent d'établir une certaine chronologie entre tous ces textes, je me contenterai ici d'exposer les conclusions auxquelles je suis arrivée. ${ }^{9}$

Alain Grandbois a vraisemblablement commencé son travail par la rédaction, sous forme de souvenirs autobiographiques, de l'anecdote du diamant disparu, comme en font foi [“Un matin à Cannes..."] et deux des manuscrits intitulés "Le diamant" (trente-trois feuillets et vingt-huit feuillets). Dans [ "Un matin à Cannes..."] et "Le diamant" de trente-trois feuillets, on trouve I'histoire de la découverte de la Côte d'Azur par Grandbois et la vie du barman de Juan-les-Pins, lequel avait été son entraîneur de natation. Progressivement, Grandbois resserre l'action autour de l'aventure de la bague perdue. Puis l'idée lui vient d'attribuer l'aventure à un personnage, Julius, ce qui lui permet de se détacher de la stricte autobiographie tout en évoquant les années d'avant-guerre et la vie insouciante et heureuse d'alors. L'invention de Julius, double de l'auteur, lance ce dernier dans une activité fébrile. On a droit alors à toute une série d'aventures à Paris, à Montréal, à Cannes ou ailleurs. Avec le personnage de Julius apparaît aussi le style sans ponctuation, style qu'on ne trouve nulle part ailleurs dans la prose de Grandbois. Cependant, l'auteur finit par revenir à son anecdote de départ. Toutefois, ses intentions se sont modifiées entre temps. Ce n'est plus tant l'histoire du diamant qui l'intéresse que

9 Chantal Bouchard, «Julius ou le double d'Alain Grandbois", Littératures, Montréal, no 3, mai 1989. 
l'évocation de la douceur de vivre des années trente et de la belle jeunesse enfuie. II abandonne donc le titre retenu jusquelà, "Le diamant", et il ajoute au récit une longue introduction où il se moque férocement et avec une certaine amertume de l'époque où il écrit.

On aurait donc, dans l'ordre de la rédaction, d'abord les textes autobiographiques, puis l'ensemble des aventures de Julius, puis les textes donnant une ébauche de l'introduction de la nouvelle, enfin ["Heureuse jeunesse..."], avant d'en arriver aux manuscrits dactylographiés qui représentent l'aboutissement du travail.

II reste les proses poétiques de "Délivrance du jour» et des autres textes de cette catégorie, impossibles à situer avec précision, ainsi que le poème “Enfer-ciel». Tout ce qu'on peut en dire est qu'ils sont postérieurs aux versions autobiographiques du "Diamant". Ils peuvent avoir été écrits après ou avant l'achèvement de la nouvelle. Toutefois, ["Les jours étaient longs..."] prose poétique également, est contemporain d'un texte intitulé "Julius et Madeleine", puisqu'il a été écrit sur la même tablette, un texte commençant à la page 10 de l'autre.

Cette chronologie relative ayant été établie, le problème qui se posait ensuite était de savoir comment on rendrait compte de tout ce dossier dans l'édition critique des nouvelles de Grandbois. Bien évidemment, il était exclu de publier intégralement la totalité des textes, à cause des contraintes d'espace, mais aussi parce que plusieurs d'entre eux sont tout à fait incomplets, voire fragmentaires, ou à peine ébauchés. Les seuls textes que nous pouvions traiter en versions, avec les variantes par rapport au texte de base, soit l'édition de $\mathrm{HMH}$, étaient le manuscrit dactylographié et sa copie au carbone, intitulés "Julius". Ce que nous fîmes.

Par ailleurs, il nous paraissait intéressant et utile, puisque pour une fois nous disposions de tant d'éléments, de donner le plus d'informations possible sur l'élaboration du texte. C'était véritablement une occasion unique, car on possède rarement plus d'un ou deux manuscrits pour les nouvelles de Grandbois. Si une édition critique doit servir à quelque chose, outre de donner des textes soigneusement établis, c'est bien, nous semblait-il, à faciliter l'étude du travail d'élaboration et des méthodes d'un auteur. Nous avons donc résolu de publier 
intégralement en annexe les versions antérieures directement liées à la nouvelle, soit [Heureuse jeunesse..."], les deux versions dactylographiées de l'introduction et le texte autobiographique intitulé "Le diamant", qui tous présentent l'anecdote de base. Nous donnons en outre in extenso le poème "Enfer-ciel», dont je n'ai guère parlé ici, mais qui illustre bien la nature d'alter ego de l'auteur du personnage nommé Julius.

Tous les autres textes, les aventures de Julius et les proses poétiques relatent des histoires très différentes de la nouvelle éditée. Nous ne pouvions donc pas les présenter comme des ébauches de celle-ci, même s'ils sont associés à son élaboration. Nous avons choisi d'en donner la description et le résumé, qui figureront également en annexe.

La présentation sous cette forme condensée de l'abondant avant-texte de "Julius" a, selon nous, l'avantage d'offrir au lecteur un bon aperçu du travail de l'écrivain. Elle donne aussi les matériaux qui permettront d'autres analyses que la nôtre sans confronter le lecteur au chaos originel.

\section{Annexe: Dossier «Julius»}

\section{Versions éditées:}

Avant le chaos, collection «L'Arbre", Montréal, HMH, 1964, p.227-241.

Cahiers de l'Académie canadienne-française, coll. "Contes et nouvelles", no 4, Montréal, 1959, p.57-67.

\section{Versions:}

"Julius", manuscrit dactylographié de 18 feuillets $(215 \mathrm{~mm} x$ $278 \mathrm{~mm}$ ), corrections à l'encre bleue et au crayon bleu, indications typographiques au crayon rouge. BNQ, 204/3/10.

"Julius", copie au carbone du précédent, corrections à l'encre bleue, les pages 13 et 15 manquent. BNQ, 204/3/10.

\section{Versions antérieures:}

[Heureuse jeunesse...], manuscrit de 20 feuillets $(127 \mathrm{~mm} \times$ $202 \mathrm{~mm})$, paginés 39-59, sans titre, encre bleue. BNQ, 204/3/10.

introduction "Le diamant", manuscrit dactylographié de 3 feuillets " $(215 \mathrm{~mm} \times 278 \mathrm{~mm})$, corrections à l'encre bleue. BNQ, 204/3/10. 
"Le diamant", copie au carbone du précédent, corrections à l'encre bleue. BNQ, 204/3/10.

autobiographique «Le diamant", manuscrit de 28 feuillets; 5 feuillets $(124 \mathrm{~mm} \times 203 \mathrm{~mm})$, paginés $I-V$, suivis de 23 feuillets $(127 \mathrm{~mm} \times 203 \mathrm{~mm})$, papier vert, paginés IV-IX (VII/7, VIII/8, IX/9) et 10-27, crayon à mine. BNQ, 204/3/10.

\section{Textes apparentés:}

autobiographique "Le diamant", manuscrit de 33 feuillets, BNQ, 204/3/10.

autobiographique [Un matin à Cannes...], manuscrit de 8 feuillets, BNQ, 204/3/10.

les aventures de Julius "Julius", manuscrit de 73 feuillets, BNQ,204/3/10.

[Tu as giflé Bernard...], manuscrit de 23 feuillets, BNQ, 204/3/16.

"Julius et Madeleine", manuscrit de 15 feuillets, le texte commence sur la page 10 d'un autre texte: [Les jours étaient longs...], BNQ 204/3/9.

"Le retour de Julius", manuscrit de 6 feuillets, BNQ, 204/3/10.

"L'Argent", manuscrit de 2 feuillets, BNQ, 204/3/10.

prose poétique "Délivrance du jour", manuscrits de 5 feuillets, 10 feuillets, 12 feuillets, 2 feuillets et 2 feuillets, BNQ, 204/3/4.

[Je puis affirmer...], manuscrit de 7 feuillets, BNQ, 204/3/10. 204/3/10.

[Julius: les fontaines...], manuscrit de 4 feuillets, BNQ,

[Les jours étaient longs...], manuscrit de 10 feuillets, BNQ, 204/3/9.

poème "Enfer-ciel", manuscrit de 6 feuillets, BNQ, 204/1/5.

\section{Chronologie probable:}

1. Textes autobiographiques

2. Aventures de Julius

[Les jours étaient longs...] et peut-être les autres proses poétiques

3. Versions antérieures

4. Versions 